\title{
Viabilidade econômica da produção de abóbora cabotiá para agricultura familiar em Iporá, Goiás
}

\author{
Economic feasibility of japanese pumpkin production for family agriculture in Iporá, Goiás \\ Viabilidad económica de la producción de calabaza cabotiá para la agricultura familiar en Iporá, \\ Goiás
}

Recebido: 19/02/2021 | Revisado: 27/02/2021 | Aceito: 06/03/2021 | Publicado: 14/03/2021

\author{
Ana Maria Cardozo Maffei \\ ORCID: https://orcid.org/0000-0002-1048-4948 \\ Instituto Federal de Educação, Ciência e Tecnologia Goiano, Brasil \\ E-mail: anamariamaffei27@outlook.com \\ Estenio Moreira Alves \\ ORCID: https://orcid.org/0000-0002-2208-7384 \\ Instituto Federal de Educação, Ciência e Tecnologia Goiano, Brasil \\ E-mail: estenio.moreira@ifgoiano.edu.br \\ Najla Kauara Alves do Vale \\ ORCID: https://orcid.org/0000-0002-3577-1513 \\ Instituto Federal de Educação, Ciência e Tecnologia Goiano, Brasil \\ E-mail: najla.vale@ifgoiano.edu.br \\ Romano Roberto Valicheski \\ ORCID: https://orcid.org/0000-0002-9623-1385 \\ Instituto Federal de Educação, Ciência e Tecnologia Goiano, Brasil \\ E-mail: romano.roberto@ifgoiano.edu.br
}

\begin{abstract}
Resumo
A análise da viabilidade econômica é imprescindível devido a sua utilização como parâmetro para a correta tomada de decisão. Assim, objetivou-se analisar a viabilidade econômica da produção de abóbora cabotiá no município de IporáGoiás, devido à sua relevância socioeconômica na realidade da agricultura familiar local. Desta maneira, elaborou-se o custo de produção e as receitas da cultura durante um ciclo para as cultivares 'Japonesa F1', 'Kyoto', 'HC 01' e 'HC 02'. Posteriormente, determinou-se de acordo com as ferramentas da análise de viabilidade econômica, o Valor Presente Líquido (VPL), a Taxa Interna de Retorno (TIR) e o Payback. Os resultados da viabilidade econômica foram determinados a partir do desempenho agronômico dos cultivares. As ferramentas VPL, TIR e o Payback apresentaram-se positivas, verificando-se a viabilidade do investimento em ambas as cultivares. Todavia, os resultados demonstram que dentre as cultivares analisadas, a que mais se destacou foi Híbrido Experimental ' $\mathrm{HC} 01$ ' na produtividade, com isto o custo de produção foi $0,56 \mathrm{R} \$ / \mathrm{Kg}$, o VPL foi positivo de $\mathrm{R} \$ 2.457,75$, a TIR foi de $18,67 \%$ a.m e o Payback foi de 3 meses.
\end{abstract}

Palavras-chave: Tomada de decisão; Custo de produção; Diversificação.

\begin{abstract}
The economic viability analysis is essential due to its use as a parameter for the correct decision making. The objective of this study was to analyze the economic viability of japanese pumpkin production in the region of Iporá, Goiás, due to its socioeconomic relevance, based on local family farming. In this way, the cost of production and crop income during a cycle for the cultivars 'Japonesa F1', 'Kyoto', 'HC 01' and 'HC 02' were elaborated. Afterwards, the Net Present Value (NPV), the Internal Rate of Return (IRR) and the Payback were determined according to the tools of economic feasibility analysis. The results of the economic viability were determined from the agronomic performance of the cultivars. The NPV, IRR and Payback tools were positive, verifying the feasibility of the investment in both cultivars. However, the results show that among the analyzed cultivars, the most outstanding was 'HC 01 ' in terms of productivity, cost of production $0.56 \mathrm{R} \$ / \mathrm{kg}$, NPV was positive of $\mathrm{R} \$ 2,457.75$, the IRR was $18,67 \%$ per month and Payback for 3 months.
\end{abstract}

Keywords: Decision making; Production cost; Diversification.

\section{Resumen}

El análisis de la viabilidad económica es fundamental por su uso como parámetro para la correcta toma de decisiones. Así, el objetivo fue analizar la viabilidad económica de la producción de calabaza cabotiá en el municipio de IporáGoiás, dada su relevancia socioeconómica en la realidad de la agricultura familiar local. De esta manera, el costo de producción y los ingresos de los cultivos se elaboraron durante un ciclo para los cultivares 'Japanese F1', 'Kyoto', 'HC 
01' y 'HC 02'. Posteriormente, se determinó el Valor Actual Neto (VAN), la Tasa Interna de Retorno (TIR) y el Payback de acuerdo con las herramientas de análisis de viabilidad económica. Los resultados de viabilidad económica se determinaron a partir del comportamiento agronómico de los cultivares. Las herramientas VPL, TIR y Payback fueron positivas, verificando la viabilidad de la inversión en ambos cultivares. Sin embargo, los resultados demuestran que entre los cultivares analizados, el que más se destacó fue el Experimental Híbrido 'HC 01' en productividad, con esto el costo de producción fue de $0,56 \mathrm{R} \$$ / Kg, el VAN fue positivo de $\mathrm{R} \$ 2.457,75$, la TIR fue del 18,67\% mensual y la recuperación de la inversión fue de 3 meses.

Palabras clave: Toma de decisiones; Costo de producción; Diversificación.

\section{Introdução}

As abóboras e as morangas são espécies pertencentes à família Cucurbitáceae, gênero Cucurbita, seu cultivo tem uma abrangência mundial, são utilizadas na alimentação humana e animal. São alimentos fonte de fósforo, ferro, vitaminas do complexo B, cálcio, provitaminas A, retinol e carboidratos. Do cruzamento entre linhagens selecionadas de morangas e abóboras, desenvolveu-se no Japão a "Tetsukabuto", cuja tradução significa “capacete de ferro", devido seus frutos apresentar coloração de cor verde escura e formato arredondado (Queiroga et al., 2017).

A abóbora híbrida apresenta grande aceitação no mercado, isso se justifica devido à sua precocidade, coloração e uniformidade no tamanho dos frutos, polpa enxuta, sabor agradável e pela diversidade no uso culinário, a qual é utilizada no preparo de saladas, sopas, pudins, refogados e bolos. Sua semente pode ser torrada e servida como aperitivo. As brotações das folhas podem ser preparadas refogadas, sendo conhecida por "cambuquira" em algumas regiões. Se armazenadas em locais fresco e seco, as abóboras maduras podem ser estocadas no pós-colheita por mais de 3 a 4 meses (Sediyama et al., 2009), característica essa importantíssima para o sucesso do produtor.

As cucurbitáceas de modo geral são de adaptadas a regiões quentes e com alta luminosidade. Em regiões de clima tropical e com baixa altitude, o plantio pode ser feito o ano todo. Esta cultura possui um amplo acesso aos canais de comercialização, podendo ser comercializadas em feiras livres, supermercados, quitandas, programas governamentais (PAA e PNAE), e nos canais de distribuições como: CEASA, os quais podem ser ofertados os frutos in natura (inteiro) ou mesmo processados em cortes e embalados (Queiroga et al., 2017; Sediyama et al., 2009).

Dentre as culturas potenciais, a abóbora cabotiá têm grande potencial de produção pelos agricultores familiares. No estado de Goiás a agricultura familiar é responsável pela complementação no fornecimento de alimentos e para a Microrregião de Iporá, as pequenas propriedades representam um dinamismo para a economia local, gerando renda e emprego para os pequenos produtores locais (Medina, 2016).

Em 2016 foram comercializados por meio das Centrais de Abastecimento do Estado de Goiás (CEASA-GO), 22.341,82 toneladas de abóbora japonesa (cabotiá), das quais 65,58\% foram produzidas no próprio estado e os demais estados tiveram uma participação de 34,32\% do total comercializado, e gerou uma receita superior a 34 milhões de reais. O município de Iporá participou apenas com 0,69\% do total de abóbora japonesa comercializadas no Ceasa - GO em 2016 (Ceasa, 2016).

Vinculado à escolha do canal de comercialização competitivo, está à escolha de um mecanismo de coordenação, que promova o desenvolvimento da atividade econômica. Uma forma de aumentar a competitividade é selecionar mecanismos de coordenação que permitam a análise do investimento realizado. Ribeiro e Silva (2008) ressaltam sobre a relevância de estudos que objetivam avaliar a viabilidade econômica das atividades agropecuárias pautada na realidade dos produtores familiares, uma vez que fornecem informações que norteiam a seleção das atividades mais rentáveis para ser desenvolvida por estes.

Portanto para obter uma avaliação assertiva da viabilidade econômica de um projeto é necessário conhecer as ferramentas de gestão financeira, cuja finalidade é auxiliar na tomada de decisão, ao qual justifique ou não o produtor investir no projeto (Santos et al., 2018). Silva e Zonin (2016) relatam que "essas ferramentas poderiam auxiliar o produtor a controlar e manejar sua propriedade e tomar decisões mais precisas com maior garantia de sucesso em seu empreendimento". 
A análise de investimento se caracteriza como um norteador no qual demonstra a real situação de um projeto, evitando o investimento de forma errônea, fazendo-se crucial no auxílio na tomada de decisão, objetivando redução de risco. Assim, objetivou-se analisar a viabilidade econômica da produção de abóbora cabotiá no município de Iporá, Goiás, voltada para a realidade local da agricultura familiar, com a intenção de verificar se é viável ou não a referida atividade. Ferramentas estas que poderão serem utilizadas pelos produtores na tomada de decisão quanto à implantação desta cultura.

\section{Metodologia}

A coleta de dados desenvolvida neste estudo foi realizada por meio do levantamento de dados primários, através das informações dos custos de produção da atividade que foi implantada em campo, as informações obtidas auxiliaram no desenvolvimento da pesquisa.

No primeiro momento da pesquisa realizou-se a coleta de dados secundários através de pesquisa bibliográfica em periódicos, livros, circulares técnicas, boletins e sites institucionais, objetivando a conceituação da temática abordada. Além disso, utilizou-se do uso da base de dados do Instituto Federal Goiano - Campus Iporá, dos resultados de pesquisa do projeto "Resgate e avaliação de variedades locais de abóbora e milho em sistemas de base agroecológica consorciados com guandu e crotalária" financiado pelo MCTI/MAPA/CNPq e seus respectivos subprojetos, a fim de avaliar os resultados produtivos do cultivo de abóbora cabotiá na localidade.

Como o objetivo deste trabalho é representar a realidade dos pequenos produtores do município de Iporá-GO, tomouse como base os dados fitotécnicos apresentados por Alves et al., (2017). No segundo momento realizou-se a coleta de dados primários, por meio do levantamento de preço dos insumos, mediante visitação "in loco" em lojas agropecuárias do município, apurando a média dos valores de cada produto.

Os dados obtidos foram submetidos à tabulação por meio da utilização do software de planilha de cálculo. Posteriormente, os dados foram alocados conforme sua classificação em custos fixos e variáveis. O tratamento dos dados da análise de viabilidade econômica utilizou-se das ferramentas de avaliação, Valor Presente Líquido, Taxa Interna de Retorno e Payback.

O Quadro 1 apresenta os itens que compõem o custo de produção (investimentos iniciais) para implantação de um projeto de produção de abóboras cabotiá por hectare, no município de Iporá. 
Quadro 1. Descrição dos custos iniciais envolvidos na produção de cabotiá em Iporá, Goiás.

\begin{tabular}{|c|c|c|c|c|c|}
\hline \multicolumn{6}{|c|}{ CUSTO DE PRODUÇÃO - 1 Hectare } \\
\hline \multicolumn{3}{|l|}{ CULTURA: Abóbora Cabotiá } & \multicolumn{3}{|c|}{ COLHEITA: Janeiro de 2015} \\
\hline \multicolumn{3}{|l|}{ PROPRIEDADE: Fazenda Escola } & \multicolumn{3}{|c|}{ MUNICÍPIO: Iporá-GO } \\
\hline \multicolumn{6}{|c|}{ CUSTOS VARIÁVEIS - INSUMOS } \\
\hline DESCRIÇÃO & QUANTIDADE & Un. & VALOR UNITÁRIO & VALOR TOTAL & $\%$ \\
\hline Calcário & 3 & ton & $\mathrm{R} \$ 105,00$ & $\mathrm{R} \$ 315,00$ & $5,01 \%$ \\
\hline Superfosfato Simples & 360 & $\mathrm{Kg}$ & $\mathrm{R} \$ 0,94$ & $\mathrm{R} \$ 338,40$ & $5,38 \%$ \\
\hline Cloreto de Potássio & 70 & $\mathrm{Kg}$ & $\mathrm{R} \$ 1,32$ & $\mathrm{R} \$ 92,40$ & $1,47 \%$ \\
\hline Ureia & 80 & $\mathrm{Kg}$ & $\mathrm{R} \$ 1,53$ & $\mathrm{R} \$ 122,40$ & $1,95 \%$ \\
\hline Sulfato de amônio & 100 & $\mathrm{Kg}$ & $\mathrm{R} \$ 1,18$ & $\mathrm{R} \$ 118,00$ & $1,88 \%$ \\
\hline Esterco curtido & 4 & $\mathrm{~m}^{3}$ & $\mathrm{R} \$ 100,00$ & $\mathrm{R} \$ 400,00$ & $6,36 \%$ \\
\hline Herbicida Dessecante & 4 & $\mathrm{~L}$ & $\mathrm{R} \$ 20,00$ & $\mathrm{R} \$ 80,00$ & $1,27 \%$ \\
\hline Sacaria para abóbora & 500 & Un & $\mathrm{R} \$ 0,20$ & $\mathrm{R} \$ 100,00$ & $1,59 \%$ \\
\hline Sementes de Abóbora Cabotiá & 0,50 & $\mathrm{Kg}$ & $\mathrm{R} \$ 1.200,00$ & $\mathrm{R} \$ 600,00$ & $9,54 \%$ \\
\hline Substrato (Mudas)* & 4 & $\mathrm{sc}$ & $\mathrm{R} \$ 5,00$ & $\mathrm{R} \$ 20,00$ & $0,32 \%$ \\
\hline Copos $(80 \mathrm{ml})$ & 25 & cento & $\mathrm{R} \$ 15,00$ & $\mathrm{R} \$ 375,00$ & $5,96 \%$ \\
\hline \multicolumn{4}{|l|}{ SUBTOTAL INSUMOS } & $\mathrm{R} \$ 2.561,20$ & $40,72 \%$ \\
\hline \multicolumn{6}{|c|}{ CUSTOS VARIÁVEIS - SERVIÇOS } \\
\hline DESCRIÇÃO & QUANTIDADE & Un. & VALOR UNITÁRIO & VALOR TOTAL & $\%$ \\
\hline Aplicação do calcário & 1,5 & $\mathrm{H} / \mathrm{M}$ & $\mathrm{R} \$ 150,00$ & $\mathrm{R} \$ 225,00$ & $3,58 \%$ \\
\hline Abertura de covas & 4,0 & $\mathrm{D} / \mathrm{H}$ & $\mathrm{R} \$ 90,00$ & $\mathrm{R} \$ 360,00$ & $5,72 \%$ \\
\hline Semeio em copos & 1,0 & $\mathrm{D} / \mathrm{H}$ & $\mathrm{R} \$ 90,00$ & $\mathrm{R} \$ 90,00$ & $1,43 \%$ \\
\hline Incorporação de adubos & 1,0 & $\mathrm{D} / \mathrm{H}$ & $\mathrm{R} \$ 90,00$ & $\mathrm{R} \$ 90,00$ & $1,43 \%$ \\
\hline Distribuição de adubos & 4,0 & $\mathrm{D} / \mathrm{H}$ & $\mathrm{R} \$ 90,00$ & $\mathrm{R} \$ 360,00$ & $5,72 \%$ \\
\hline Transplantio & 2,0 & $\mathrm{D} / \mathrm{H}$ & $\mathrm{R} \$ 90,00$ & $\mathrm{R} \$ 180,00$ & $2,86 \%$ \\
\hline $1^{\circ}$ adubação de cobertura & 1,0 & $\mathrm{D} / \mathrm{H}$ & $\mathrm{R} \$ 90,00$ & $\mathrm{R} \$ 90,00$ & $1,43 \%$ \\
\hline $2^{\circ}$ adubação de cobertura & 1,0 & $\mathrm{D} / \mathrm{H}$ & $\mathrm{R} \$ 90,00$ & $\mathrm{R} \$ 90,00$ & $1,43 \%$ \\
\hline Aplicação herbicida & 1,0 & $\mathrm{D} / \mathrm{H}$ & $\mathrm{R} \$ 120,00$ & $\mathrm{R} \$ 120,00$ & $1,91 \%$ \\
\hline Colheita/Acondicionamento & 8,0 & $\mathrm{D} / \mathrm{H}$ & $\mathrm{R} \$ 90,00$ & $\mathrm{R} \$ 720,00$ & $11,45 \%$ \\
\hline Montagem do sistema de irrigação & 2,0 & $\mathrm{D} / \mathrm{H}$ & $\mathrm{R} \$ 90,00$ & $\mathrm{R} \$ 180,00$ & $2,86 \%$ \\
\hline \multicolumn{4}{|l|}{ SUBTOTAL SERVIÇOS } & $\mathbf{R} \$ \mathbf{2 . 5 0 5 , 0 0}$ & $\mathbf{3 9 , 8 3 \%}$ \\
\hline \multicolumn{6}{|c|}{ CUSTOS FIXOS } \\
\hline DESCRIÇÃO & QUANTIDADE & Un. & VALOR UNITÁRIO & VALOR TOTAL & $\%$ \\
\hline Sistema de Irrigação por Gotejamento & 1 & ciclo & $\mathrm{R} \$ 589,55$ & $\mathrm{R} \$ 589,55$ & $9,37 \%$ \\
\hline Custo de Oportunidade da Terra (IGP-M) & 1 & $\begin{array}{l}\text { ha/ } \\
\text { ciclo }\end{array}$ & $\mathrm{R} \$ 144,79$ & $\mathrm{R} \$ 144,79$ & $2,30 \%$ \\
\hline Projeto Técnico & 1 & - & $\mathrm{R} \$ 129,09$ & $\mathrm{R} \$ 129,09$ & $2,05 \%$ \\
\hline Visitas Técnicas & 3 & visitas & $\mathrm{R} \$ 120,00$ & $\mathrm{R} \$ 360,00$ & $5,72 \%$ \\
\hline \multicolumn{4}{|l|}{ SUBTOTAL FIXOS } & $\mathrm{R} \$ \mathbf{1 . 2 2 3 , 4 3}$ & $19,45 \%$ \\
\hline \multicolumn{4}{|c|}{ CUSTO TOTAL POR HECTARE } & $\mathrm{R} \$ 6.289,63$ & $100,00 \%$ \\
\hline
\end{tabular}


Nota: Valores atualizados em Iporá em 2018.

* Produzido na propriedade.

Fonte: Autores.

Os custos foram estimados a partir de investimentos em sistema de irrigação por gotejamento com pressão por gravidade, insumos (sementes), sacaria, calcário, transplantio, adubações de cobertura, abertura de covas, dentre outros.

O projeto foi baseado de acordo com os coeficientes técnicos de produção obtidos através dos resultados de pesquisa descritos por Alves et al., (2017). A semeadura foi realizada de no dia 31 de outubro de 2014, foram utilizados blocos casualizados, composto por quatro tratamentos (híbridos) e quatro repetições. As mudas foram transplantadas para covas espaçadas em 2,0 x 2,5 m, apresentando um estande de 2000 plantas por hectare.

O preço para determinar o custo fixo da terra foi baseado no preço descrito pelo ANUALPEC 2015, no qual o valor inicial é R\$ 5.433,00 / ha em Iporá e regiões circunvizinhas. O valor da terra foi corrigido com base no Índice Geral de Preços do Mercado (IGP-M) até o início do período correspondente ao ciclo produtivo da cultura. Posteriormente com o valor corrigido obteve-se o preço do hectare da terra de R \$ 5.577,79. Determinou-se o custo de oportunidade do capital investido na terra com base na inflação do período do ciclo produtivo, totalizando R\$ 144,79 (Quadro 2). O custo de elaboração do projeto técnico foi estimado em R $\$ 129,09$.

Quadro 2. Inflação dos meses de 2014 e 2015, através do IGP-M.

\begin{tabular}{|l|c|}
\hline \multicolumn{2}{|c|}{ IGP-M entre 2014 - 2015 } \\
\hline Meses & Percentual no mês (\%) \\
\hline Outubro de 2014 & $0,28 \%$ \\
\hline Novembro de 2014 & $0,98 \%$ \\
\hline Dezembro de 2014 & $0,62 \%$ \\
\hline Janeiro de 2015 & $0,76 \%$ \\
\hline
\end{tabular}

Fonte: http://www.portalbrasil.net/igpm.htm (2017).

O custo de oportunidade do capital foi baseado no valor de $\mathrm{R} \$ 7.737,40$, corrigido com base no Índice Nacional de Preços ao Consumidor - Amplo (IPCA), o valor do capital corrigido foi de R $\$ 7.968,03$, descontou-se deste o valor inicial, resultando em $\mathrm{R} \$ 230,63$ (Quadro 3).

Quadro 3. Inflação dos meses de 2014 e 2015, através do IPCA.

\begin{tabular}{|l|c|}
\hline \multicolumn{2}{|c|}{ IPCA entre 2014 - 2015 } \\
\hline Meses & Percentual no mês (\%) \\
\hline Outubro de 2014 & $0,42 \%$ \\
\hline Novembro de 2014 & $0,51 \%$ \\
\hline Dezembro de 2014 & $0,78 \%$ \\
\hline Janeiro de 2015 & $1,24 \%$ \\
\hline
\end{tabular}

Fonte: http://www.portalbrasil.net/ipca.htm (2017). 
O presente estudo foi realizado abrangendo um recorte de quatro meses correspondente ao ciclo produtivo das cultivares. Para esta simulação foi considerado que o investimento inicial foi realizado com recursos próprios do empreendedor.

\section{Critérios de Avaliação de Investimentos}

A análise da viabilidade econômica evidencia-se como fator preponderante, no que tange a tomada de decisão de se fazer ou não o investimento em determinado projeto (Begiato; Meneghini, 2015). As ferramentas da análise da viabilidade econômica são: Valor Presente Líquido Eq. (1), Taxa Interna de Retorno Eq. (2) e Payback Eq. (3)

$$
V P L=\sum_{j=1}^{n} \frac{F C_{j}}{(1+i)^{n}}-F C_{0}
$$

Onde: $\boldsymbol{F} \boldsymbol{C}_{\boldsymbol{J}}$ : representa um valor de entrada ou saída num determinado tempo; $\boldsymbol{F} \boldsymbol{C}_{\mathbf{0}}$ : fluxo de caixa no momento zero, no momento inicial (investimento). De acordo com Castelo Branco (2010), cita os critérios para se aceitar o VPL: Se o VPL for $=0$, então o projeto não proporciona lucro ou prejuízo; Se o VPL for maior, se aceita o projeto; Se o VPL for menor, rejeita-se o projeto.

Assaf Neto e Lima (2014) define a fórmula da Taxa Interna de Retorno:

$$
F C_{0}=\sum_{j=1}^{n} \frac{F C_{J}}{(1+i)^{J}}
$$

Onde: $\boldsymbol{F} \boldsymbol{C}_{0}=$ Valor do fluxo no momento zero (investimento); $\boldsymbol{F} \boldsymbol{C}_{\boldsymbol{j}}=$ Fluxos de caixas futuros de entradas e saídas; $\boldsymbol{i}$ = taxa de desconto ou taxa interna de retorno, iguala em determinado tempo as entradas e saídas previstas.

Para Gitman (2010), os critérios de decisão quando utilizamos o período de Payback (3) para aceitar ou rejeitar um projeto, é: Se o período de Payback for < do que o período de Payback máximo aceitável, então se aceita o projeto. Se o período de Payback for > do que o período de Payback máximo aceitável, então se rejeita o projeto.

A partir dos resultados obtidos, foi realizada a análise da viabilidade econômica da produção de híbridos de cabotiás no município de Iporá, GO. Desse modo, foi elaborado um fluxo de caixa, o qual considera os investimentos (saídas de caixa) e receitas (entradas de caixa), como receitas foram consideradas todas as entradas obtidas oriundas da comercialização da produção. Posteriormente, a elaboração dos fluxos de caixa, analisou-se o Valor Presente Líquido (VPL), a Taxa Interna de Retorno (TIR) e o Payback para as cultivares híbridos de cabotiás: 'Japonesa F1', 'Kyoto', 'HC 01' e 'HC 02'.

\section{Resultados e Discussão}

A estimativa de investimento na produção das cultivares dos híbridos de 'Japonesa F1', 'Kyoto', 'HC 01' e 'HC 02', conforme demonstrado Tabela 1, foi de $\mathrm{R} \$ 6.289,63$, cerca de 36,25\% do custo total de produção é composto por investimento de custeio com mão-de-obra. Portanto, esta atividade possui vocação à agricultura familiar, podendo ser mitigada por meio da utilização de mão-de-obra familiar, aumentando a receita do produtor, contribuindo para a geração de divisas para a localidade, 
distribuição de renda e fixação de homem no campo, bem como, a geração de empregos.

$\mathrm{Na}$ Tabela 1, é apresentado a produtividade obtida para cada cultivar. Observa-se que os híbridos experimentais em desenvolvimento pelo Instituto Federal Goiano - Campus Iporá alcançou maiores produtividade que os híbridos comerciais. Todavia, como os híbridos 'HC 01 'e 'HC 02' sejam experimentais, e não estejam disponíveis no mercado, dentre os híbridos comerciais o produtor deverá escolher prioritariamente o cultivar 'Kyoto' em detrimento da 'Japonesa F1'. Quando comparado ao híbrido 'Japonesa F1', a produtividade dos híbridos 'HC 01', 'HC 02' e 'Kyoto', foi 28,45\%, 26,41\%, 11,20\% superior respectivamente (Tabela 1), proporcionando em menor custo de produção.

Tabela 1. Produtividade de frutos comerciais (Kg/ha) de cultivares híbridos de cabotiá, Iporá-Goiás, 2015.

\begin{tabular}{lr}
\hline Cultivares & Produtividade (Kg/ha) \\
\hline Japonesa F1 & $8.753,75$ \\
Kyoto & $9.735,00$ \\
HC 01 IF Goiano & $11.245,00$ \\
HC 02 IF Goiano & $11.066,25$
\end{tabular}

Fonte: Alves et al. (2017).

A Tabela 2 demonstra o custo de produção em $\mathrm{R} \$ / \mathrm{Kg}$ para cada cultivar, considerando que as culturas 'HC 01' e 'HC 02' foram mais produtivas, seus custos de produção $\mathrm{R} \$ / \mathrm{Kg}$ foram os menores, sendo respectivamente $\mathrm{R} \$ 0,56$ e $\mathrm{R} \$ 0,57$.

Já para os híbridos comerciais 'Japonesa F1' e 'Kyoto' obteve um custo de produção de R $\$ 0,72$ e R $\$ 0,65$ por quilo de abóbora, respectivamente. Resultados estes, se devem a menor produtividade que onera o custo de produção (Tabela 1). Utilizando a cultivar 'Japonesa F1' como parâmetro para mensurar o custo por unidade ( $\mathrm{R} \$ / \mathrm{Kg}$ ) produzida para as demais cultivares, visualizando-se que para as cultivares 'Kyoto', 'HC 01' e 'HC 02' o custo foi inferior a 'Japonesa F1' em 9,73\%, $22,23 \%, 20,84 \%$ respectivamente.

Até que os híbridos experimentais estejam disponíveis no mercado a escolha entre os cultivares 'Japonesa F1' e 'Kyoto', a melhor escolha fica será o cultivar híbrido 'Kyoto', pois, apresenta menor custo por unidade produzida e maior produtividade.

Tabela 2. Custo de produção dos cultivares híbridos de cabotiá (R\$/kg), Iporá, Goiás, 2015.

\begin{tabular}{lcr}
\hline Cultivares & Custo $(\mathrm{R} \$ / \mathrm{Kg})$ & $\%$ \\
\hline Japonesa F1 & $\mathrm{R} \$ 0,72$ & $100 \%$ \\
Kyoto & $\mathrm{R} \$ 0,65$ & $90,27 \%$ \\
HC 01 IF Goiano & $\mathrm{R} \$ 0,56$ & $77,77 \%$ \\
HC 02 IF Goiano & $\mathrm{R} \$ 0,57$ & $79,16 \%$ \\
\hline
\end{tabular}

Fonte: Autores.

O preço adotado para a comercialização no momento da colheita foi estipulado de acordo com as cotações do CEASA - GO no pós-colheita, adotando-se o valor médio de R\$1,15 por quilo de abóbora. Ressalta-se, que os preços praticados no mercado local são superiores devido ao custo do frete da CEASA-GO até o mercado local. O que melhora o cenário para o produtor local e o torna mais competitivo.

O custo unitário de outras regiões é muito similar (Biancardi et al., 2017), reforçando a capacidade competitiva dos 
produtores que se voltam ao mercado local. A estratégia de abastecimento com produção local corrobora muito para a redução da pegada ambiental proporcionada pelo transporte de alimentos em nosso país continental (Azevedo, 2015).

Conforme Tabela 3, a maior receita bruta da comercialização da produção foi para a cultivar 'HC 01' (R \$12.931,75); e para a cultivar 'HC 02' (R\$12.657,19). Já para os híbridos 'Japonesa F1' e 'Kyoto', obteve-se menor renda de (R\$10.066,01) e (R\$11.195,25), respectivamente.

Tabela 3. Receita Total advinda da estimativa de comercialização da produção das cultivares no momento da colheita, Iporá, Goiás, 2015.

\begin{tabular}{lccc}
\hline Cultivares & Produtividade $(\mathrm{Kg} / \mathrm{ha})$ & Preço de Venda $(\mathrm{R} \$ / \mathrm{kg})$ & $\begin{array}{c}\text { Receita Total } \\
(\mathrm{R} \$)\end{array}$ \\
\hline Japonesa F1 & 8753,75 & $\mathrm{R} \$ 1,15$ & $\mathrm{R} \$ 10.066,81$ \\
Kyoto & 9735,00 & $\mathrm{R} \$ 1,15$ & $\mathrm{R} \$ 11.195,25$ \\
HC 01 IF Goiano & 11245,00 & $\mathrm{R} \$ 1,15$ & $\mathrm{R} \$ 12.931,75$ \\
HC 02 IF Goiano & 11006,25 & $\mathrm{R} \$ 1,15$ & $\mathrm{R} \$ 12.657,19$ \\
\hline
\end{tabular}

Fonte: Autores.

Vale ressaltar que, a comercialização da produção foi destinada para o abastecimento do comércio local. Portanto, não contabilizados na análise econômica os custos com frete. Ressalta-se ainda, que os custos com atravessadores e logística, oneram muito para aos produtores de outras regiões, propiciando assim, mais competitividade aos produtores locais.

Neste estudo foi adotada a taxa mínima de atratividade de 8,69\% a.m, sendo o percentual da taxa Selic (Sistema Especial de Liquidação e de Custódio) acumulada no período vigente de outubro de 2014 a janeiro de 2015 de 3,69\% (Tabela 4), época da coleta de dados do estudo, acrescido da liquidez do capital investido de 5\%.

Tabela 4. Percentual da taxa Selic entre os meses de outubro de 2014 a janeiro de 2015.

\begin{tabular}{lcr}
\hline Mês & Mensal & Acumulada no período \\
\hline Outubro de 2014 & $0,95 \%$ a.m & $0,95 \%$ \\
Novembro de 2014 & $0,84 \%$ a.m & $1,79 \%$ \\
Dezembro de 2014 & $0,96 \%$ a.m & $2,75 \%$ \\
Janeiro de 2015 & $0,94 \%$ a.m & $3,69 \%$ \\
\hline
\end{tabular}

Fonte: Ministério da Fazenda.

O fluxo de caixa foi elaborado (Quadro 4), de acordo com o ciclo produtivo da cultura, ou seja, quatro meses abrangendo desde o manejo inicial de solo (preparo) até o momento da colheita e comercialização. Estão descritos no fluxo de caixa todos os investimentos ocorridos durante o ciclo produtivo da cultura e as receitas advindas da comercialização da produção.

Considerando a produtividade obtida em um hectare, conforme Tabela 1, para o híbrido 'Kyoto', ao final do ciclo produtivo, obteve-se uma receita de R \$11.195,25, proveniente da comercialização de $9.735 \mathrm{Kg} / \mathrm{ha}$. Já para a cultivar 'HC 01' obteve uma receita de $\mathrm{R} \$ 12.931,75$, advindos da produção em um hectare. A produtividade foi de $11.245 \mathrm{Kg} / \mathrm{ha}$ e para a cultivar 'HC 02', obteve uma receita de R\$ 12.657,19, advindos da produção de 11.006,25 Kg/ha. Quanto a cultivar 'Japonesa F1' a receita foi de $\mathrm{R} \$ 10.066,81$. 
Quadro 4. Fluxo de caixa de produção das cultivares 'Japonesa 1', 'Kyoto', 'HC 01' e 'HC 02'.

\begin{tabular}{|l|l|r|r|r|r|}
\hline Cultivares & \multicolumn{1}{|c|}{ Mês } & \multicolumn{1}{c|}{0} & \multicolumn{1}{c|}{1} & \multicolumn{1}{c|}{3} \\
\hline \multirow{4}{*}{ Japonesa F1 } & Investimento & $-\mathrm{R} \$ 6.597,40$ & $-\mathrm{R} \$ 300,00$ & $-\mathrm{R} \$ 120,00$ & $-\mathrm{R} \$ 820,00$ \\
\cline { 2 - 6 } & Receitas & $\mathrm{R} \$ 0,00$ & $\mathrm{R} \$ 0,00$ & $\mathrm{R} \$ 0,00$ & $\mathrm{R} \$ 10.066,81$ \\
\cline { 2 - 6 } & Fluxo de Caixa & $-\mathrm{R} \$ 6.597,40$ & $-\mathrm{R} \$ 300,00$ & $-\mathrm{R} \$ 120,00$ & $\mathrm{R} \$ 9.246,81$ \\
\hline \multirow{3}{*}{ Kyoto } & Investimento & $-\mathrm{R} \$ 6.597,40$ & $-\mathrm{R} \$ 300,00$ & $-\mathrm{R} \$ 120,00$ & $-\mathrm{R} \$ 820,00$ \\
\cline { 2 - 6 } & Receitas & $\mathrm{R} \$ 0,00$ & $\mathrm{R} \$ 0,00$ & $\mathrm{R} \$ 0,00$ & $\mathrm{R} \$ 11.195,25$ \\
\cline { 2 - 6 } & Fluxo de Caixa & $-\mathrm{R} \$ 6.597,40$ & $-\mathrm{R} \$ 300,00$ & $-\mathrm{R} \$ 120,00$ & $\mathrm{R} \$ 10.375,25$ \\
\hline \multirow{3}{*}{$\mathrm{HC} 01$ IF Goiano } & Investimento & $-\mathrm{R} \$ 6.597,40$ & $-\mathrm{R} \$ 300,00$ & $-\mathrm{R} \$ 120,00$ & $-\mathrm{R} \$ 820,00$ \\
\cline { 2 - 6 } & Receitas & $\mathrm{R} \$ 0,00$ & $\mathrm{R} \$ 0,00$ & $\mathrm{R} \$ 0,00$ & $\mathrm{R} \$ 12.931,75$ \\
\cline { 2 - 6 } & Fluxo de Caixa & $-\mathrm{R} \$ 6.597,40$ & $-\mathrm{R} \$ 300,00$ & $-\mathrm{R} \$ 120,00$ & $\mathrm{R} \$ 12.111,75$ \\
\hline \multirow{3}{*}{ HC 02 IF Goiano } & Investimento & $-\mathrm{R} \$ 6.597,40$ & $-\mathrm{R} \$ 300,00$ & $-\mathrm{R} \$ 120,00$ & $-\mathrm{R} \$ 820,00$ \\
\cline { 2 - 6 } & Receitas & $\mathrm{R} \$ 0,00$ & $\mathrm{R} \$ 0,00$ & $\mathrm{R} \$ 0,00$ & $\mathrm{R} \$ 12.657,19$ \\
\cline { 2 - 6 } & Fluxo de Caixa & $-\mathrm{R} \$ 6.597,40$ & $-\mathrm{R} \$ 300,00$ & $-\mathrm{R} \$ 120,00$ & $\mathrm{R} \$ 11.837,19$ \\
\hline
\end{tabular}

Fonte: Autores.

A partir dos dados apresentados no fluxo de caixa, calculou-se o Valor Presente Líquido, Taxa Interna de Retorno e o Payback. A priori para qualquer tomada de decisão em que projeto investir é a análise da viabilidade econômica, no que tange, ao setor rural que é cercado por diversos gargalos que influenciam no sucesso do empreendimento, estas tornam-se essenciais. $\mathrm{O}$ atual cenário econômico, não permite o investimento errôneo, fazendo-se necessário à utilização das ferramentas de viabilidade econômica.

Para calcular o VPL, desconta-se a soma de todos os valores futuros de fluxo de caixa menos a taxa mínima de atratividade $(8,69 \%)$. O VPL se mostra positivo para todas os cultivares. Assim, apresenta retorno superior ao percentual mínimo esperado de $(8,69 \%)$ adotado.

O cultivar que obteve o VPL mais expressivo foi a 'HC 01 ' de $\mathrm{R} \$ 2.457,75$ conforme Tabela 5, justificando o investimento nesta cultura produtiva, uma vez que, a cultura gera uma rentabilidade superior a taxa mínima de atratividade, sendo este um critério de referência pelo qual se define se um projeto deve ou não ser aceito.

Para as cultivares 'HC 02', 'Kyoto' e 'Japonesa F1', o VPL foi positivo e gerou um excedente de R\$ 2.243,92, R\$ $1.105,35$ e $\mathrm{R} \$ 226,51$ respectivamente. O valor presente líquido é uma das ferramentas mais sofisticadas dos métodos de análise de viabilidade econômico, pois esta considera a valorização do dinheiro no tempo.

A diferença de VPL das cultivares comerciais 'Japonesa F1' e 'Kyoto' é de R\$ 878,84 (487,99\%), ou seja, a escolha de investimento da cultivar 'Kyoto' em detrimento da 'Japonesa F1' é cerca de 5 vezes superior de rentabilidade de acordo com $\mathrm{o}$ indicador VPL. Para as demais cultivares 'HC 01'e 'HC 02' em comparativo com a 'Japonesa F1', apresenta um VPL superior a 'Japonesa F1' em 1.085,04\% e 990,64\% respectivamente. 
Tabela 5. Indicadores de viabilidade econômica dos cultivares de cabotiá, Iporá, Goiás, 2015.

\begin{tabular}{lccc}
\hline Cultivares & Payback & VPL & TIR \\
\hline Japonesa F1 & 3 meses & R $\$ 226,51$ & $9,17 \%$ a.m \\
Kyoto & 3 meses & R $\$ 1.105,35$ & $13,10 \%$ a.m \\
HC 01 IF Goiano & 3 meses & R $\$ 2.457,75$ & $18,67 \%$ a.m \\
HC 02 IF Goiano & 3 meses & R $\$ 2.243,92$ & $17,83 \%$ a.m \\
\hline
\end{tabular}

Fonte: Autores.

No que diz respeito ao Payback todas as cultivares obtiveram um Payback de 3 meses, conforme a Tabela 5. O saldo do Payback no mês de liquidação do investimento foi de $\mathrm{R} \$ 2.229,41$; R 2.257,85; e R\$ 5.094,35 e R\$ 4.819,70 para as cultivares 'Japonesa F1', 'Kyoto', 'HC 01' e 'HC 02', respectivamente.

A taxa interna de retorno foi superior a TMA para todas as cultivares, apresentando os seguintes resultados $9,17 \%$, $13,10 \% ; 18,67 \%$ e 17,83\% respectivamente para as cultivares 'Japonesa F1', 'Kyoto', 'HC 01' e 'HC 02', indicando a viabilidade destes híbridos, uma vez que há agregação de valor ao investimento.

A TIR da cultivar 'HC 01 ' foi de $18,67 \%$ a.m superior à taxa mínima atratividade $(8,69 \%)$, ou seja, houve um lucro sobre a TMA de 9,98\%, indicando que é mais atrativo investir na cultura 'HC 01', do que aplicar o mesmo capital em uma aplicação financeira a uma taxa de $(8,69 \%)$.

A partir das análises de custo de produção, das receitas e a viabilidade econômica das cultivares 'japonesa F1', 'Kyoto', 'HC 01' e 'HC 02', verificou-se que todas as cultivares justifica-se o investimento, uma vez que, sob a análise resultou em um VPL positivo, Payback positivo e a TIR positiva, obtendo a viabilidade da implantação de qualquer uma das cultivares no município estudado.

Para todas as cultivares o Payback ou prazo do retorno do investimento foram de 3 meses, ou seja, o Payback ocorreu no momento da comercialização da produção e consequentemente pós ciclo produtivo das cultivares. A partir da análise da ferramenta TIR, observou-se que todas as cultivares obtiveram uma taxa interna de retorno superior a TMA, ou seja, para as condições determinísticas apresentadas o investimento em qualquer uma das cultivares é mais lucrativo do que investir o capital em aplicação financeira.

Em termos comparativos, de acordo com os indicadores de viabilidade econômica para as cultivares analisadas 'Japonesa F1', 'Kyoto', 'HC 01' e 'HC 02' foram respectivamente a ferramenta VPL de R\$ 226,51, R\$ 1.105,35, R\$ 2.457,75 e R\$2.243,92. A taxa interna de retorno demonstrou que a cultivar que possibilita mais rentabilidade é a 'HC 01'é a TIR de 18,67\%, e para as cultivares 'Japonesa F1', 'Kyoto' e 'HC 02' foi de 9,17\%, 13,10\% e 17,83\% respectivamente.

Embora a TIR seja inferior aos apresentados por Leite et al (2016), nota-se que o Payback é muito inferior. O curto período necessário para recuperar o dinheiro investido na atividade é fundamental para o sucesso da agricultura familiar (Costa et al., 2005). Vale ressaltar que embora a TIR seja menor da abobricultura, a flexibilidade em permitir alteração da atividade é fundamental frente a dinâmica do mercado. Em se tratando da hidroponia descrita por Leite et al. (2016) somente no quarto ano atinge-se o Payback, fortemente influenciado pelo auto investimento inicial desta atividade.

Logo, a produção de abóbora tem grande potencial para diversificação e investimento nos solos das propriedades. A correção e manutenção da capacidade produtiva é fundamental para a permanência dos agricultores familiares nas propriedades. Uma vez que, recuperar solos degradados pode refletir em Payback de oito anos conforme descritos por Costa et al. (2005) em sistema composto pelo cultivo de arroz e melão.

Portanto, o investimento em preparo de solo e fertilidade empregados no cultivo da abóbora poderão ser utilizados por outras culturas sucessoras. Tem-se, portanto, saldo em serviços agronômicos não contabilizados que serão disponibilizados a 
propriedade/produtores.

Os híbridos possuem um nicho de mercado diversificado, devido ao seu uso culinário, estando presente em diversos pratos, onde seu sabor e coloração são atrativos aos mais diversos paladares. Outro fator importante é que por serem frutos resistentes, possibilita a sua armazenagem por um período de 4 meses pós colheita; propiciando ao produtor a opção pela comercialização em períodos de melhores preços de mercado. O cultivo dessa atividade propicia também melhoria na fertilidade do solo, devido à quantidade de insumos aplicados na área.

A utilização das ferramentas de viabilidade econômica faz-se necessário, uma vez que, evidencia possíveis riscos e incertezas de investimentos errôneos. O processo de coleta de dados, organização do fluxo de caixa, custos, despesas e cálculos dos indicadores podem ser utilizados como ferramentas para a tomada de decisão, para a aplicação em outros munícipios considera-se as variações das condições do mercado regional.

O estudo propõe mais uma ferramenta para orientar os produtores que possuam o interesse em investir nesta cultura, fornecendo instrumentos que possibilitam ter maior competitividade de mercado, além, de servir de aporte de base dados de futuras pesquisas relacionadas à temática abordada.

\section{Conclusão}

Conclui-se que, o cultivar mais atrativo ao investimento no que tange a retornos econômicos foi o híbrido experimental do IF Goiano 'HC 01'. Entretanto, por não estar disponível ainda para comercialização, recomenda-se dentre os híbridos comerciais o híbrido 'Kyoto', uma vez que este obteve a TIR e VPL, superior a 'Japonesa F1'.

Conclui-se ainda que a análise quanto mais profunda, proporciona melhores respostas, e que as ferramentas VPL, TIR e Payback corroboram para definir se será ou não viável investir na cultura. Ressalta-se que o investimento nesta atividade, se justifica pelo ciclo curto da cultura que é de aproximadamente 4 meses, possibilitando o exercício de outra atividade na mesma área, além de ser uma excelente opção de diversificação da produção. Sugere-se que futuros trabalhos avalie os efeitos da sazonalidade de oferta e das estações produtivas ao longo do ano sobre o desempenho produtivo e viabilidade econômica.

\section{Agradecimentos}

Agradecemos as instituições CNPq (Processo 473115/2014-0) pelo auxílio financeiro concessão de bolsas e ao Instituto Federal Goiano, Campus Iporá. Agradecemos aos servidores, estudantes e colaboradores sem os quais não seria possível a realização deste trabalho.

\section{Referências}

Alves, E. M., Santos, L. J., Batista, G. S., Souza, S. R., Claudio, F. L., Calgaro Junior, G., Santos, L. C., \& Paim, T. P. (2017). Características e produtividade de híbridos experimentais de abóbora "japonesa” em Iporá - GO. PUBVET, 11: 662-669. http://DX.DOI.ORG/10.22256/PUBVET.V11N7.662-669

ANUALPEC. (2015). Anuário da Pecuária Brasileira.

Assaf Neto, A. (2009). Matemática financeira e suas aplicações. Editora, Atlas.

Azevedo, E. (2015). O ativismo alimentar na perspectiva do Locavorismo. Ambiente e Sociedade, 18(3): 81-98. http://dx.doi.org/10.1590/18094422ASOC740V1832015

Begiato, G. F., \& Meneghini, R. C. M. (2015). Oportunidade de investimento em agroindústria de beneficiamento de Castanha-do-Brasil. Custos $e$ @ gronegócio on line, 11: 13-55. http://www.custoseagronegocioonline.com.br/numero2v11/K\%202\%20investimento.pdf

Biancardi, C. C. S., Rodrigues, R., Siqueira, H. M., Carneiro, J. J., \& Fernandes, M. A. (2017). Viabilidade econômica da produção familiar de alimentos para os mercados institucionais de Alegre-ES. Extensão Rural, 23(2): 85-101. http://dx.doi.org/10.5902/2318179621669

Castelo Branco, A. C. (2010). Matemática financeira aplicada: método algébrico, HP-12C, Microsoft Excel@. 
Research, Society and Development, v. 10, n. 3, e23410313110, 2021

(CC BY 4.0) | ISSN 2525-3409 | DOI: http://dx.doi.org/10.33448/rsd-v10i3.13110

CEASA-GO. (2016). Central de Abastecimento do Estado de Goiás- Ceasa - GO. Análise Conjuntural. Goiânia: CEASA-GO, 2016. < http://www.sgc.goias.gov.br/upload/arquivos/2017-08/anAlise-conjuntural-2016_compressed.pdf >.

Costa, R. N. T., Oliveira Junior, N. O., Saunders, L. C. U., \& Biserra, J. V. (2005). Indicadores econômicos da recuperação de um solo sódico em condições de drenagem subterrânea no Vale do Curu, CE. Irriga, 10(3): 272-278. http://dx.doi.org/10.15809/irriga.2005v10n3p272-278

Gitman, L. J. (2010). Princípios de administração financeira. Pearson Prentice Hall.

IGP-M (2017). Índice Geral de Preços do Mercado. http://www.portalbrasil.net/igpm.htm

IPCA. (2017). Índice Nacional de Preço ao Consumidor Amplo. http://www.portalbrasil.net/ipca.htm

Leite, D., Migliavacca, R. A., Alfredo Junior, L. A. M., Albrecht, P., \& Fausto, D. A. (2016). Viabilidade econômica da implantação do sistema hidropônico para alface com recursos do PRONAF em Matão-SP. Revista iPecege, 2(1): 57-65. http://dx.doi.org/10.22167/r.ipecege.2016.1.57

Medina, G. (2016). Agricultura familiar em Goiás: Lições para o assessoramento técnico. (3a ed.) Rev. e Ampl. Goiânia, GO, Brasil.

Queiroga, R. C. F., Silva, G. D., Pereira, A. M., Almeida, R. R. P., \& Silva, A. B. (2017). Yield and quality of the Tetsukabuto squash fruits induced with 2,4D doses under dry conditions. Horticultura Brasileira, 35(2): 271-277. http://dx.doi.org/10.1590/s010205362017021

Ribeiro, S. C., \& Silva, M. L. (2008). Viabilidade econômica de atividades florestais e agropecuárias. Revista Política Agrícola, 2: 76-85. https://seer.sede.embrapa.br/index.php/RPA/article/viewFile/410/361

Santos, G., Botelho, F. J., Meneghel, J. M. M., \& Fausto, D. A. (2018). Resultado econômico de confinamento de bovinos de corte em diferentes cenários. Revista Ipecege, 4(3): 15-22. https://doi.org/10.22167/r.ipecege.2018.3.15

Sediyama, M. A. N., Vidigal, S. M., Santos, M. R. dos., \& Mascarenhas, M. H. T. (2009). Cultura da moranga híbrida ou abóbora Tetsukabuto. (EPAMIG, Boletim Técnico, 92). Belo Horizonte, MG, Brasil.

Silva, V. K., \& Zonin, V. J. (2016). Gestão em propriedades rurais em transição agroecológica nos municípios de São Carlos e Águas de Chapecó-SC. Empreendedorismo, Gestão e Negócios, 5(5): 273-291. http://www.fatece.edu.br/arquivos/arquivos\%20revistas/empreendedorismo/volume5/15.pdf 